\title{
CMEARTICLE
}

\section{Clinics in diagnostic imaging (150)}

Nor Azam Mahmud ${ }^{1}$, MBBs, MMed, Dinesh $R \underline{\text { Singh }}^{2}$, MBBS, FRCR, Steven BS $\underline{\text { Wong }}^{3}$, MBChB, MMed, Wilfred CG Peh ${ }^{2}$, FRCP, FRCR
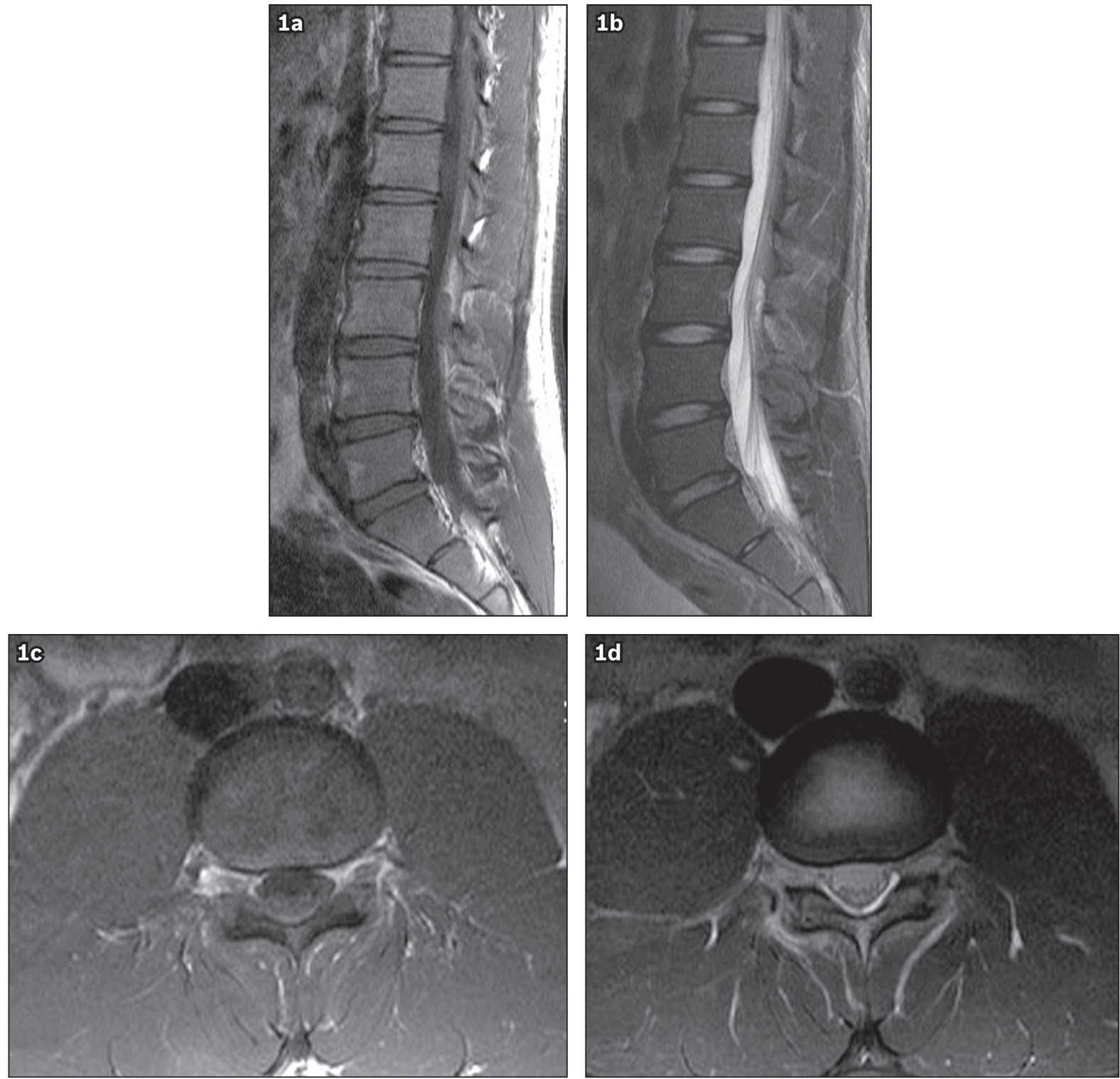

Fig. 1 Sagittal (a) T1-W and (b) T2-W MR images of the lumbar spine. Axial (c) T1-W and (d) T2-W MR images taken at the L3 vertebral level.

\section{CASE PRESENTATION}

A 19-year-old Chinese man presented to the emergency department with pain in the lower back, which started soon after he had carried some heavy boxes. He denied any fall or trauma preceding the pain. There was no sciatica or neurological deficit on examination. He maintained a normal gait and anal tone. The lumbar spine radiographs were normal. Magnetic resonance (MR) imaging was requested in order to evaluate his persistent back pain. What do the MR images (Figs. 1a-d) show? What is the diagnosis?

${ }^{1}$ Department of Diagnostic Radiology, Universiti Sains Malaysia, Kota Bharu, Malaysia, ${ }^{2}$ Department of Diagnostic Radiology, Khoo Teck Puat Hospital, Alexandra Health, ${ }^{3}$ Department of Diagnostic Radiology, Singapore General Hospital, Singapore

Correspondence: Prof Wilfred CG Peh, Professor and Head, Department of Diagnostic Radiology, Khoo Teck Puat Hospital, Alexandra Health, 90 Yishun Central, Singapore 768828.wilfred.peh@gmail.com 


\section{IMAGE INTERPRETATION}

The MR images (Fig. 1) show a small posterior epidural fluid collection at the L3 vertebral level. On sagittal T1-weighted and T2-weighted MR images (Figs. 1a \& b), a biconvex-shaped lesion in the posterior epidural space, which is hyperintense on all sequences, is observed. Axial MR images (Figs. 1c \& d) confirm that the crescentric hyperintense lesion is located in the posterior epidural space. There is associated bone marrow oedema of the adjacent L3 pedicle, inferior articular process and L4 superior articular process. Additional findings (not shown) include spina bifida occulta and an L5 pars defect.

\section{DIAGNOSIS}

Acute spontaneous spinal epidural haematoma.

\section{CLINICAL COURSE}

The patient was admitted under the care of a spinal surgeon. Management was conservative and there was gradual resolution of his back pain. No neurological deficit was detected on discharge or at subsequent follow-up one week later. Repeat MR imaging five months later (Figs. 2a-d) showed total resolution of the lumbar spinal epidural haematoma.

\section{DISCUSSION}

Spontaneous spinal epidural haematoma (SSEH) is a recognised cause of acute back pain. The pain is usually of acute or subacute onset, and may be accompanied by signs of cauda equina syndrome. ${ }^{(1)} \mathrm{A}$ history of minor or no preceding trauma is common.

Spinal epidural haematomas can be trauma-related or spontaneous. Causes of traumatic spinal epidural haematoma include vertebral fractures, postoperative haemorrhage, epidural anaesthesia-related bleed and missile injuries. ${ }^{(2)}$ Cervical spondylosis, bone disorders such as rheumatoid arthritis, Paget's disease or ankylosing spondylitis could be possible predisposing factors. ${ }^{(3)}$ Post-traumatic spinal epidural haematoma is more common in elderly male patients with pre-existing vertebral disease. In a young patient, a predisposing bone lesion is usually the underlying cause. Postsurgical prognosis is usually better in patients with no spinal fractures, as there is less underlying cord injury. ${ }^{(3)}$

No identifiable cause is found in $40 \%-50 \%$ of reported cases of SSEH. Up to $30 \%$ of cases are related to the use of anticoagulants. ${ }^{(4,5)}$ Reported predisposing factors include coagulopathy, anticoagulation, vascular anomaly, disc herniation, Paget's disease, Valsalva manoeuvre, hypertension and neoplasms. ${ }^{(1,5)}$ While SSEH is regarded as a rare entity, its diagnosis is now more frequent than before, owing to the increased availability and use of MR imaging. MR imaging is now the investigation of choice for the detection and diagnosis of SSEH.

SSEH commonly occurs in the cervicothoracic junction in patients below 40 years of age. ${ }^{(6,7)}$ A slight male bias is present. ${ }^{(4,6)}$
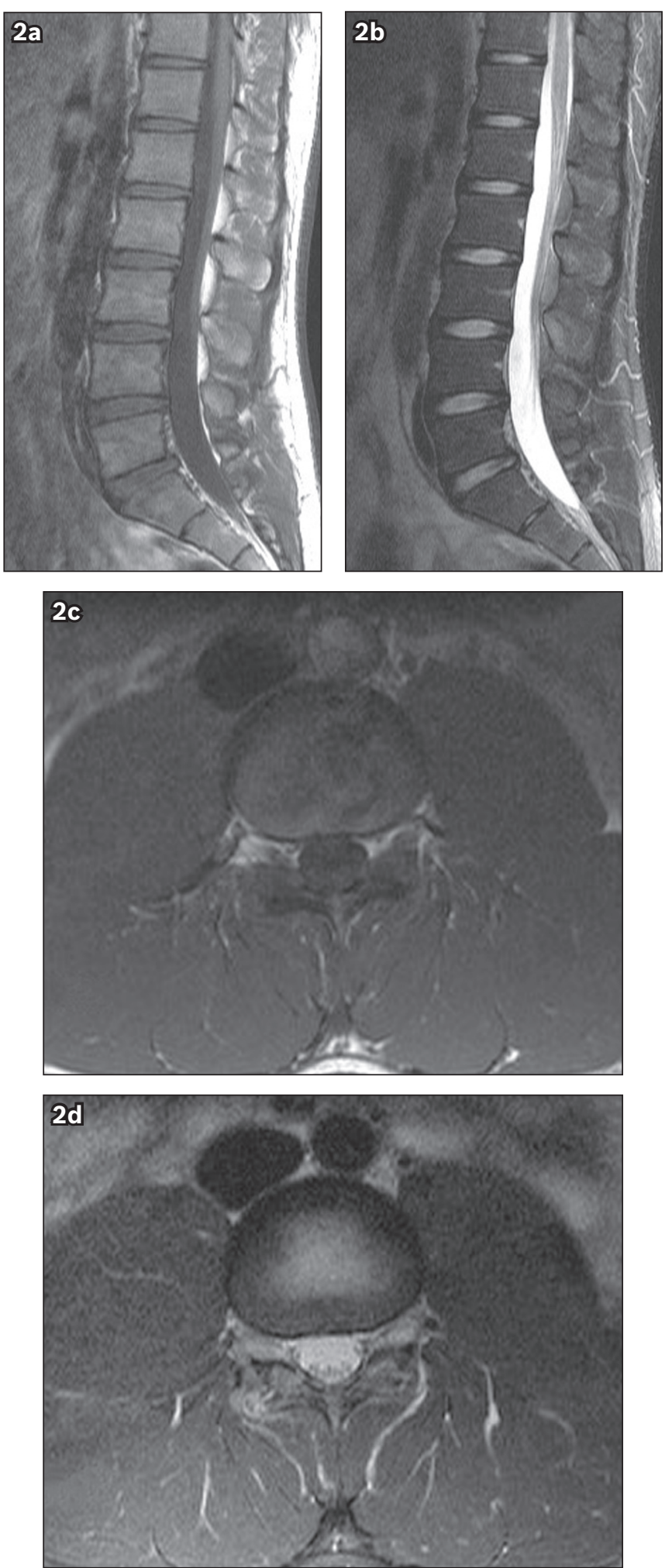

Fig. 2 Follow-up MR images obtained five months after discharge. Sagittal (a) T1-W and (b) T2-W MR images; and axial (c) T1-W and (d) T2-W MR images show total resolution of the posterior spinal epidural haematoma that was previously present at the patient's L3 vertebral level.

Those older than 40 years of age show two peak locations, namely the cervicothoracic and thoracolumbar junctions. ${ }^{(4,8)}$ Almost all SSEH lesions are located posterior to the dural sac, as the anterior aspect of the dural sac is closely apposed to the posterior longitudinal ligament of the spinal column. ${ }^{(4,9)}$ 

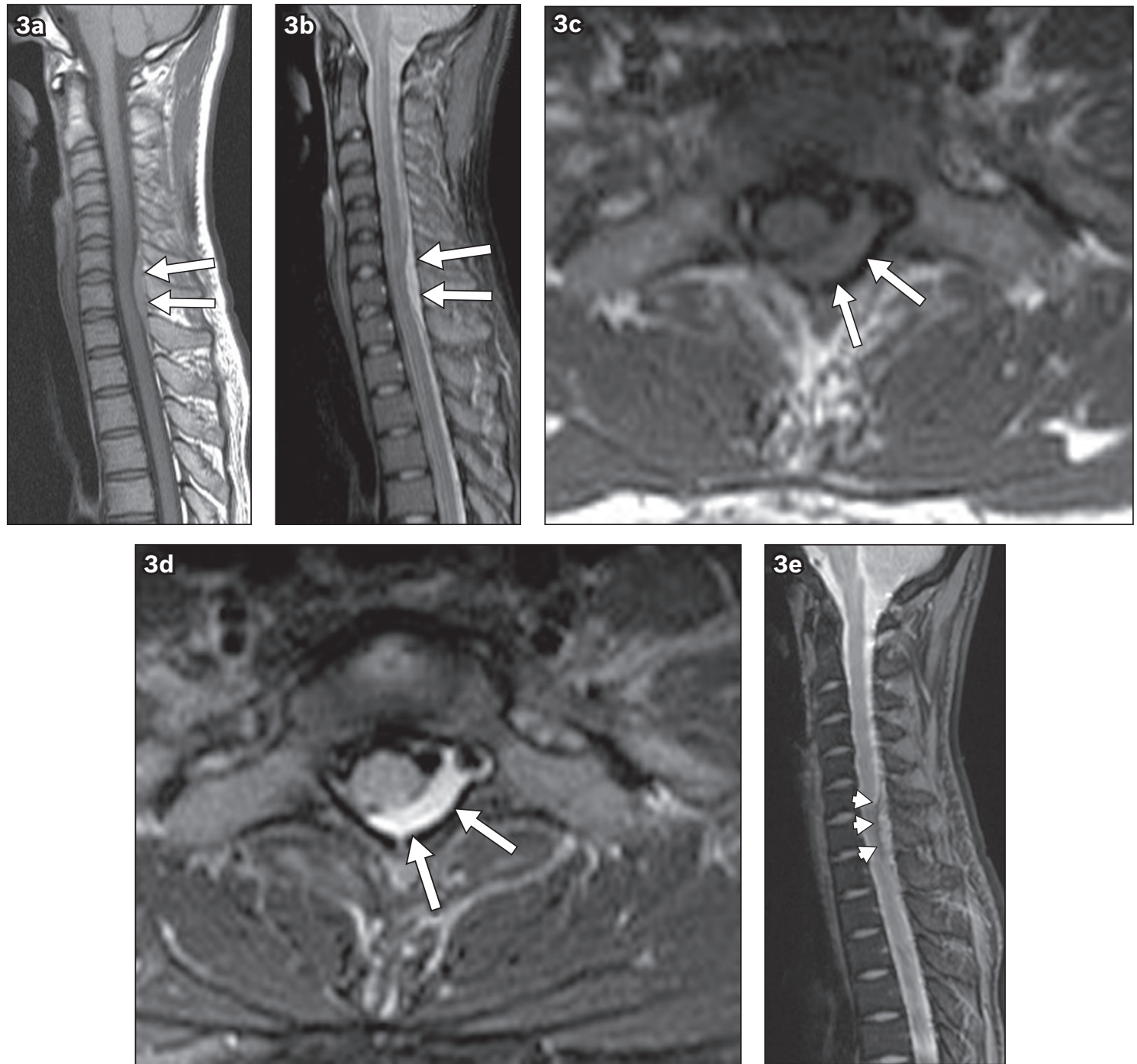

Fig. 3 A 31-year-old Chinese woman presented with acute onset of intrascapular pain. She denied any preceding trauma or fall. Clinical examination showed bilateral lower limb weakness with hypertonia, clonus, hyperreflexia and extensor plantar reflexes. (a) Sagittal T1-W MR image shows a hyperintense lesion in the posterior epidural space. The lesion extends from the C 6 to T1 levels and has a convex outline (arrows). (b) Sagittal STIR MR image shows the hyperintense lesion sited posterior to the spinal cord from the C6 to T1 levels (arrows). (c) Axial T1-W MR image taken at the C7/T1 vertebral level shows a crescentic hyperintense lesion in the left posterior spinal canal (arrows). (d) Axial T2-W MR image taken at the C7/T1 level shows a hyperintense signal in the crescentic lesion (arrows), pushing the spinal cord anteriorly and to the right. (e) Sagittal T2*-W GRE MR image shows the posterior spinal canal lesion with cerebral spinal fluid isointense signal with a hypointense anterior rim (arrowheads), thus confirming the presence of blood products. The patient underwent emergency spinal surgery, consisting of left hemilaminectomy from the C6 to T1 levels, and an epidural haemorrhage consisting of subacute stagnant blood was evacuated.

The presence of the posterior epidural venous plexus supports the commonly accepted theory of a venous cause to the haematoma, where the valveless venous plexus permits a reversal in blood flow when pressure is increased during injury or activity. ${ }^{(9)}$ Some authors have postulated that the predominance of SSEH in the posterior epidural location is due to the higher stretch and shear forces from spinal movements on the posterior epidural venous plexus than on those positioned anteriorly. ${ }^{(6)}$ An arterial cause of SSEH is based on reported cases of incidentally detected arteriovenous malformations and the observation that arterial haemorrhage is more likely to result in a pressure effect on the spinal cord, as seen in some cases of SSEH. ${ }^{(4)}$

Clinically, patients with SSEH present with acute onset of severe spinal pain, often localised with eventual radicular radiation. This commonly progresses to symptoms of cord compression or cauda equina syndrome. In most cases, the clinical course is rapid. Some patients, however, show a delay before the development of neurological deficit or even relapsing symptoms, which complicate diagnosis. ${ }^{(4,6)}$ Neurological deficits can range from mild to moderate, and can be unilateral or bilateral, depending on the location of the SSEH. Sometimes, SSEH, particularly those in the lumbar region, can mimic the symptoms of a prolapsed intervertebral disc. MR imaging, with its multiplanar capability, has replaced computed tomography myelography as the investigation of choice, thus increasing detection of SSEH.

On sagittal MR images, SSEH is seen as a biconvex-shaped mass separated from the underlying neural structures by a curvilinear low-signal-intensity structure - the dura mater 
(Figs. 3a \& b). The displaced location of the dura mater and its contents confirms the epidural location of the mass. The mass is predominantly located within the posterior epidural space, showing well-defined contours and tapering of the superior and inferior margins. ${ }^{(4,6,9)}$ On axial MR images, dural displacement is often more easily seen and the laterality of the mass is also better appreciated (Figs. 3c \& d). Assessment for intraforaminal extension of the mass is often better on axial images, as is the diagnosis of nerve root compression. The SSEH may appear concave or convex on axial images. ${ }^{(10)}$

The T1 and T2 signal intensity of SSEH depends on the age, size and oxygenation of the clot, with frequent variations seen in cases of uncertain chronology. Signal heterogeneity follows the stage of degradation of haemoglobin and is identical to that described for intracranial haemorrhage. In the hyperacute phase (i.e. within the first 24 hours), SSEH is isointense to hypointense on T1-weighted images, and hyperintense on T2-weighted images, owing to the presence of oxyhaemoglobin within fresh oxygenated blood. During the acute phase (i.e. days 1-3), progression to deoxyhaemoglobin causes the appearance of the clot to be slightly $\mathrm{T} 1$ hypointense to isointense and $\mathrm{T} 2$ hypointense. In the early subacute phase (i.e. days 3-7), the clot contains intracellular methaemoglobin, leading to marked T1-shortening, as well as T2-shortening. As a result, the clot appears hyperintense on T1-weighted images and hypointense on T2-weighted images. Fat-suppressed T1-weighted images are helpful in differentiating the T1 hyperintense SSEH from epidural fat.

In the late subacute phase (i.e. weeks 1-2), the clot is hyperintense on both T1- and T2-weighted images (Figs. 3a-d) due to extracellular methaemoglobin from the lysis of erythrocytes within the clot. Chronic SSEH, seen after two weeks, contains haemosiderin, which causes the clot to be predominantly $\mathrm{T} 1$ and $\mathrm{T} 2$ hypointense. The presence of haemosiderin is readily confirmed with gradient echo MR sequences that show hypointensity and blooming (Fig. 3e). ${ }^{(11)}$ In some cases, T2 heterogeneity is seen, usually as hypointense foci within a primarily $\mathrm{T} 2$ hyperintense lesion. This is explained by the presence of deoxyhaemoglobin or fibrous septa attaching the dura to the walls of the spinal canal. ${ }^{(1)}$

Intravenous contrast administration has reportedly resulted in varying appearances - peripheral, central, homogeneous or heterogeneous enhancement. ${ }^{(12)}$ The most common pattern is peripheral enhancement of the SSEH, postulated as being due to dural hyperaemia or thickening, ${ }^{(1)}$ or from septa or vessels in the epidural fat. ${ }^{(10)}$ Central contrast enhancement, which is less commonly seen, is deemed to be due to the extravasation of contrast-enhanced blood from leaking vessels, possibly accounting for its heterogeneous appearance. ${ }^{(5)}$ Significant central enhancement of the haematoma in the hyperacute stage may suggest active bleeding, warranting emergency spinal surgery. ${ }^{(13)}$ While intravenous contrast administration is not a requirement for the diagnosis of SSEH, it may aid in diagnosis or the exclusion of causes, which includes intraspinal malignancy such as metastases, schwannoma and lymphoma, or an arteriovenous malformation or aneurysm. ${ }^{(10)}$

SSEH is distinguishable from spinal subdural haematoma (SSH) on MR imaging, although both may present with similar presenting clinical complaints. $\mathrm{SSH}$, however, has a more rapid evolution. On MR imaging, the T1- and T2-weighted signal characteristics of SSEH and SSH may be similar. SSH shows some predominance in the thoracic and thoracolumbar spine, with few reported cases in the cervicothoracic junction. On sagittal images, SSH may have a convex configuration, but on axial images, it is more crescentic in shape, incompletely surrounding the spinal cord. The perilesional curvilinear hypointense dura mater may not be evident with $\mathrm{SSH}$ as opposed to SSEH. The inverted 'Mercedes star sign' in the lumbar region suggests a subdural location of the haematoma, while extension of the spinal haematoma into the subdural space of the posterior cranial fossa is a good indicator of $\mathrm{SSH} .{ }^{(10,11)}$ The pattern of contrast enhancement in $\mathrm{SSH}$ is similar to that seen in SSEH.

Other differential diagnoses of SSEH include epidural abscess, epidural malignancy (e.g. metastases, schwannoma, lymphoma), epidural lipoma and prolapsed intervertebral disc (Fig. 4). Epidural abscess is usually ventrally sited, involves multiple levels, and is associated with acute discitis, which should be evident on contrast-enhanced MR imaging. Epidural metastases often have vertebral infiltration and associated pathological fractures, and there is typically a known history of a primary malignancy (Fig. 5). Epidural malignancies such as schwannoma and lymphoma will not show any temporal change in T1- and T2-weighted MR imaging characteristics due to degradation of haemoglobin. This is also the case for epidural lipomas, which seldom have acute onset of symptoms. Fat-suppressed MR sequences may also help to distinguish SSEH from a lipoma. ${ }^{(9,14)}$

Treatment options for SSEH vary according to the acuteness of presentation and the severity of symptoms or neurological deficits. The widely accepted treatment for SSEH is early surgical intervention, preferably within 48 hours in patients with complete sensorimotor deficit. This usually involves posterior exposure of all the levels involved, as demonstrated on MR imaging, with laminectomy and evacuation of the haematoma. Organised clots should be removed from the dura and nerve roots, and any active bleeding points, located and cauterised. Whether insertion of subfascial drains is required is controversial and subject to the individual surgeon's preference. ${ }^{(15)}$ Surgical outcome is better for haematomas located in the lumbosacral region compared to those located in the thoracic region. ${ }^{(16)}$ Some patients, predominantly those with mild and non-progressive symptoms such as those present in our patient, may be managed conservatively, with follow-up imaging to ascertain resolution of the SSEH.(1) Reversal of coagulopathy, if present, should always be done. 

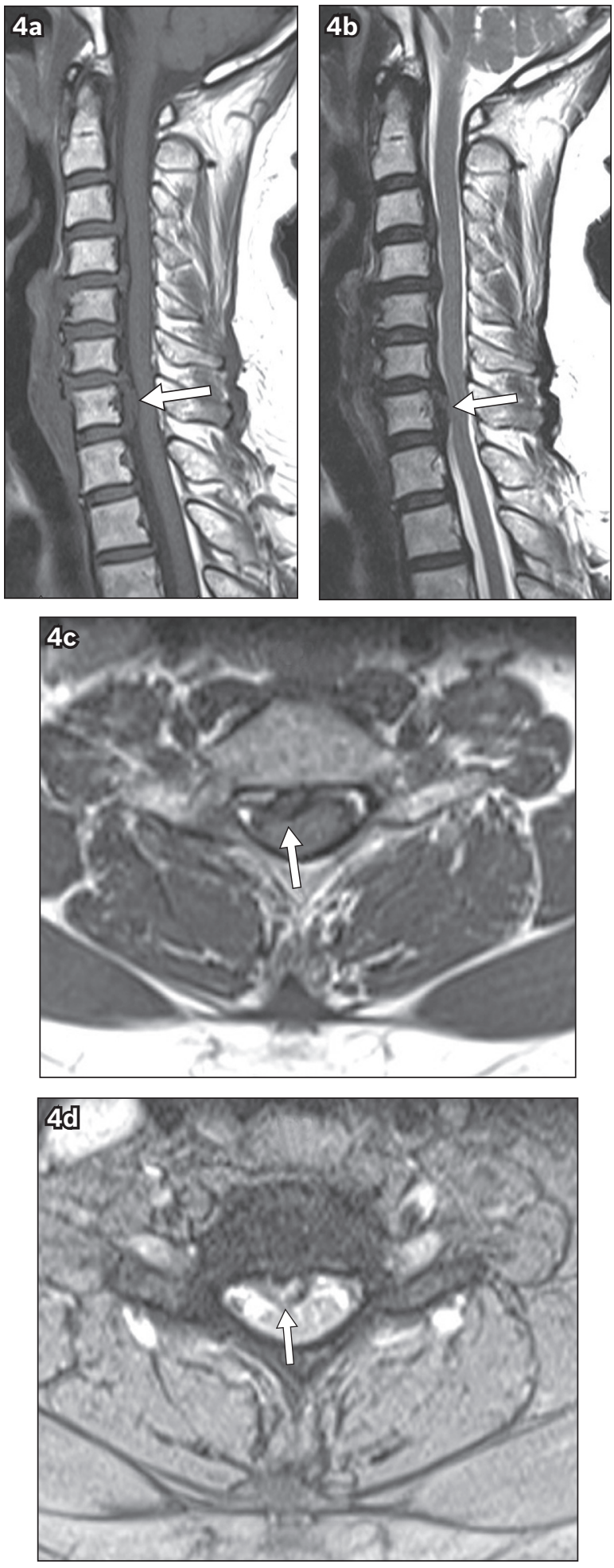

Fig. 4 Prolapsed cervical intervertebral disc in a 57-year-old Chinese woman who presented with left-sided limb weakness and numbness. Sagittal (a) T1-W and (b) T2-W MR images of the cervical spine show a largely isointense epidural mass (arrow) located posterior to the $\mathrm{C} 7$ vertebral body. It represents a cranially migrated $\mathrm{C} 7 / \mathrm{T} 1$ disc. She also had less severe disc protrusions at multiple levels. Axial (c) T1-W and (d) T2*-W GRE MR images taken at the $C 7$ level show the migrated disc, seen as an anterior epidural mass (arrow) indenting the adjacent spinal cord.

In one series, SSEH-related mortality was reported to be $5.7 \%$ and the surgery-related complication rate was $2.9 \%$, with few deaths resulting from surgery. ${ }^{(8)}$ While some studies have shown that outcomes are related to the duration of spinal
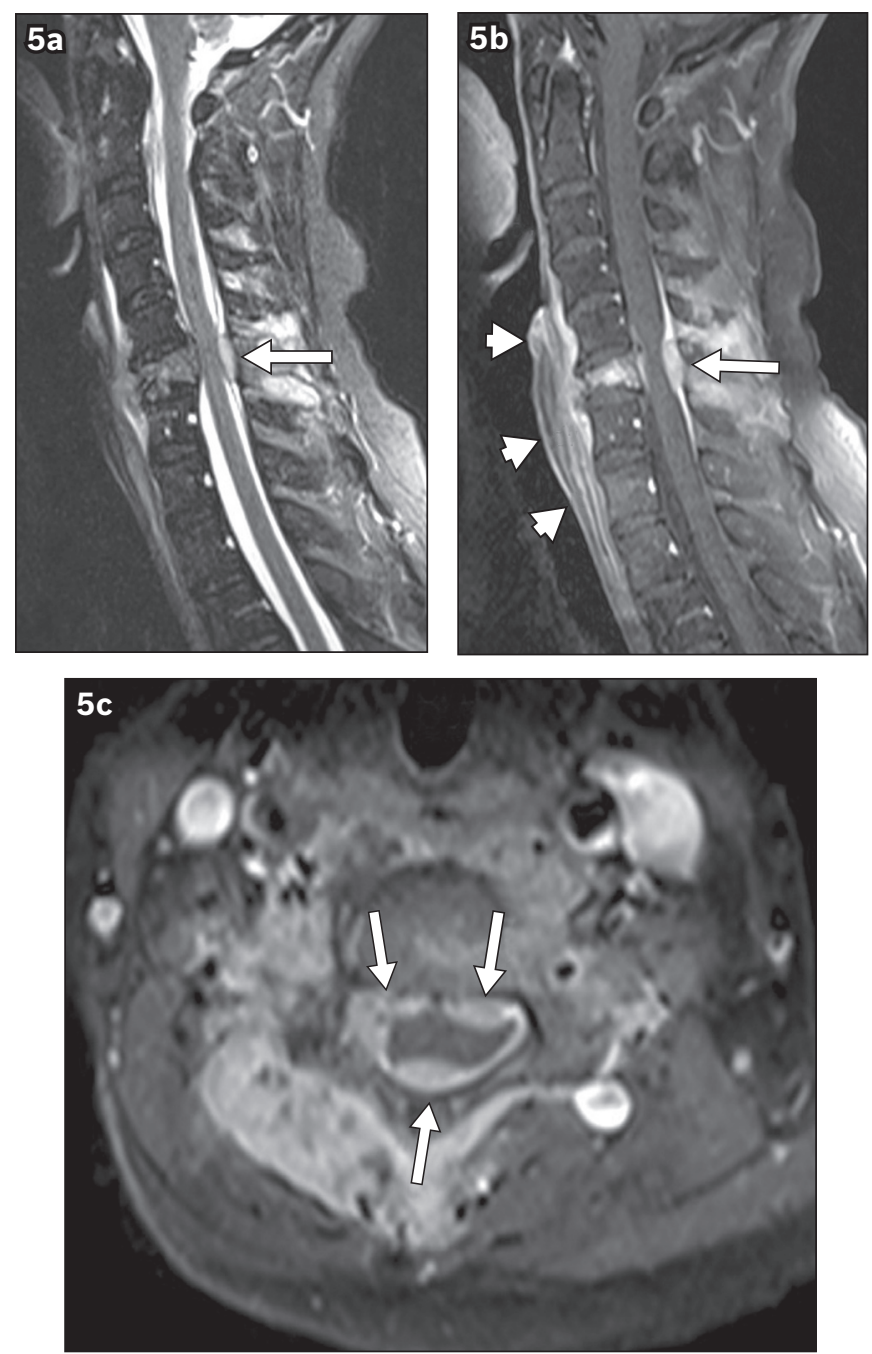

Fig. 5 Epidural metastasis in a 61-year-old Chinese woman with biopsy-proven poorly differentiated renal cell carcinoma. She presented with right arm pain. (a) Sagittal T2-W MR image shows a T2-hyperintense epidural mass (arrow) compressing the anterior and posterior aspects of the spinal cord, with abnormal increased T2-hyperintensity within the bone marrow of a compressed C6 vertebral body and the soft tissues anterior to the C6 vertebral body, as well as adjacent to the C6 spinal process. (b) Corresponding enhanced sagittal and (c) axial (taken at the C6 level) fat-suppressed T1-W MR images confirm an extensive epidural enhancing mass (arrows) encircling and compressing the thecal sac and spinal cord. There is also prominent enhancing prevertebral soft tissue (arrowheads) extending from the C5 to T1 levels, and the C6 vertebral body, as well as bilateral posterior paraspinal muscle involvement.

cord symptoms and the interval to surgery, other studies have found no difference in outcome and that a longer preoperative interval may not necessarily entail a poorer prognosis. ${ }^{(8)}$ Nonetheless, Chang et al showed that patients with severe neurological deficits prior to treatment (i.e. surgery), particularly those with autonomic dysfunction, MR findings of cord myelopathy or infarction, have poorer outcomes. Hence, it was ascertained that neurological deficit after symptom onset was more important with regard to the prognosis than the management approach. No correlation has been found between prognosis and the patient's age, the local extent of the SSEH or the segmental location. ${ }^{(9)}$ Conversely, Fukui et al stated that surgical outcome is more favourable in SSEH of 
the lumbar region and when SSEH is confined to one vertebral level. ${ }^{(1)}$

In summary, SSEH should always be considered as a potential differential diagnosis in patients presenting with acute and progressive back pain. This entity is readily diagnosed on MR imaging, and good outcomes can be expected with emergency surgery. Variations in MR imaging signal characteristics following the evolution of the haematoma should be appreciated in line with the chronology of symptoms.

ABSTRACT Spontaneous spinal epidural haematoma is a rarely encountered cause of back pain. It often leads to cauda equina syndrome, necessitating emergency spinal surgery. We report the case of a 19-year-old Chinese man who presented with pain in the lower back, which started after he had carried some heavy boxes. He denied a history of fall or trauma. Magnetic resonance (MR) imaging showed a hyperintense biconvex-shaped lesion in the posterior epidural space on both T1- and T2-weighted sequences, diagnostic of a spinal epidural haematoma. The patient, who was admitted and managed conservatively, had gradual resolution of his pain. No neurological deficit was detected on discharge or on follow-up. Repeat MR imaging showed total resolution of the lumbar spinal epidural haematoma. The clinical characteristics, MR imaging features, diagnosis and management of spontaneous spinal epidural haematoma are discussed.

Keywords: spinal emergency, spinal haemorrhage, spontaneous haemorrhage, spontaneous spinal epidural haematoma

\section{REFERENCES}

1. Fukui MB, Swarnkar AS, Williams RL. Acute spontaneous spinal epidural hematomas. AJNR AM J Neuroradiol 1999; 20:1365-72.

2. Hsieh CT, Chiang YH, Tang CT, Sun JM, Ju DT. Delayed traumatic thoracic spinal epidural hematoma: a case report and literature review. Am J Emerg Med 2007; 25:69-71.

3. Foo D, Rossier AB. Post-traumatic spinal epidural hematoma. Neurosurgery 1982; 11(1 Pt 1):25-32.

4. Dinsmore AJ, Leonard RB, Manthey D. Spontaneous spinal epidural hematoma: a case report. J Emer Med 2005; 28:423-6.

5. Chen CJ, Ro LS. Central gadolinium enhancement of an acute spontaneous spinal epidural haematoma. Neuroradiology 1996; 38 Suppl 1:S114-6.

6. Jamjoon ZA. Acute spontaneous spinal epidural hematoma: the influence of magnetic resonance imaging on diagnosis and treatment. Surg Neurol 1996; 46:345-9.

7. van Heesewijk JP, Casparie JW. Acute spontaneous spinal epidural hematoma in a child. Eur Radiol 2000; 10:1874-6.

8. Ravi D, Dwarakanath S, Satish R, Gopal S, Venkataramana NK. Spontaneous spinal extradural hematomas. J Clin Neurosci 2006; 13:269-72.

9. Chang FC, Lirng JF, Luo CB, et al. Evaluation of clinical and MR findings for the prognosis of spinal epidural haematomas. Clin Radiol 2005; 60:762-70.

10. Karadeli E, Ulu EMK, Ozdemir O, Calisaneller T. Contrast enhancement of acute spinal epidural haematoma and its potential of mimicking a tumor: case report and review of literature. Eur J Radiol Extra 2008; 65:43-6.

11. Braun P, Kazmi K, Nogués-Meléndez P, Mas-Estellés F, Aparici-Robles F. MRI findings in spinal subdural and epidural hematomas. Eur J Radiol 2007; 64:119-25.

12. Kirsch EC, Khangure MS, Holthouse D, McAuliffe W. Acute spontaneous spinal subdural hematoma: MRI features. Neuroradiology 2000; 42:586-90.

13. Nawashiro H, Higo R. Contrast enhancement of a hyperacute spontaneous spinal epidural hematoma. AJNR Am J Neuroradiol 2001; 22:1445.

14. Holtås S, Heiling M, Lönntoft M. Spontaneous spinal epidural hematoma: findings at MR imaging and clinical correlation. Radiology 1996; 199:409-13.

15. Kebaish KM, Awad JN. Spinal epidural hematoma causing acute cauda equina syndrome. Neurosurg Focus 2004; 16:e1.

16. Nayak N, Baldawa S, Diyora B, Sharma A. Delayed recovery of paraplegia following surgical evacuation of spontaneous cervicothoracic epidural hematoma. Childs Nerv Syst 2011; 27:2031-2. 


\section{SINGAPORE MEDICAL COUNCIL CATEGORY 3B CME PROGRAMME} (Code SMJ 201312B)

Question 1. Regarding spinal epidural haematoma:
(a) It could be related to trauma.
(b) It could be spontaneous.
(c) Postsurgical prognosis is better in patients with spinal fractures.
(d) Post-traumatic haematoma is more common in the elderly.

Question 2. Regarding imaging of spinal epidural haematoma:
(a) Radiography is the modality of choice.
(b) Ultrasonography has an important role.
(c) Computed tomography has no role.
(d) MR imaging is the modality of choice.

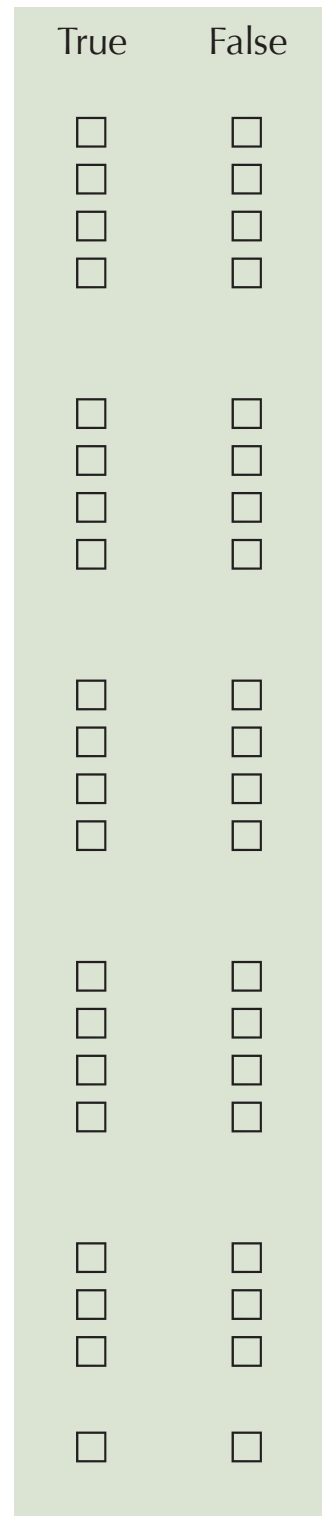

Question 3. Regarding MR imaging findings in spinal epidural haematoma:
(a) It is seen as a biconvex-shaped mass
(b) A mass is predominantly located in the anterior epidural space.
(c) Axial images are useful in assessing intraforaminal extension.
(d) It may appear concave or convex on axial images.

Question 4. Regarding MR imaging of spinal epidural haematoma:
(a) Signal heterogeneity is independent of the stage of degradation of haemoglobin.
(b) Fat-suppressed T1-weighted images help differentiate it from epidural fat.
(c) A chronic clot is predominantly $\mathrm{T} 1$ and $\mathrm{T} 2$ hypointense.
(d) Blooming may be seen on gradient echo images.

Question 5. Regarding the management of spinal epidural haematoma:
(a) Treatment options vary according to the severity of symptoms.
(b) Early surgical intervention is never indicated.
(c) Surgical outcome is usually better for haematomas located in the thoracic region than for those located in the lumbosacral region.
(d) Surgical outcome is usually better for cases with cord myelopathy or infarction.

\footnotetext{
Doctor's particulars:

Name in full

MCR number

Email address

Specialty:

SUBMISSION INSTRUCTIONS:

(1) Log on at the SMJ website: http://www.sma.org.sg/publications/smjcurrentissue.aspx and select the appropriate set of questions. (2) Provide your name, email address and MCR number. (3) Select your answers and click "Submit".

RESULTS:

(1) Answers will be published in the SMJ February 2014 issue. (2) The MCR numbers of successful candidates will be posted online at the SMJ website by 20 January 2014. (3) Passing mark is $60 \%$. No mark will be deducted for incorrect answers. (4) The SMJ editorial office will submit the list of successful candidates to the Singapore Medical Council. (5) One CME point is awarded for successful candidates.

Deadline for submission: (December 2013 SMJ 3B CME programme): 12 noon, 13 January 2014.
} 